\title{
Chapter 1 Articulation and Sharing of Distributed Design Project and Process Knowledge
}

\author{
Vadim Ermolayev, Frank Dengler, Eyck Jentzsch, and Wolf-Ekkehard \\ Matzke
}

\begin{abstract}
The paper reports on the work in the case study of the ACTIVE project on the use of the combination of knowledge process learning, articulation and sharing technologies for increasing the performance and decreasing the ramp-up efforts in engineering design projects. This knowledge is mined from distributed heterogeneous datasets, fused, and further used for visualizing design project plan information in a way that suggests optimized project plans and fosters collaboration on these knowledge structures in development teams. Software demonstrator architecture, implementation and validation are presented. Validation results indicate that the solution is effective in providing expert assistance to design project managers in performing their typical planning tasks.
\end{abstract}

\subsection{Introduction}

In knowledge economies knowledge workers [4] are central to an organizations success - yet the tools they must use often stand in the way of maximizing their productivity. A need to remedy some of the defects of current knowledge worker tools caused primarily by the requirement for greater knowledge worker productivity becomes substantially more demanded recently and across industries. Knowledge workers acting alone but more importantly in distributed teams are of a particular concern and focus in our research. One

\footnotetext{
Vadim Ermolayev · Eyck Jentzsch · Wolf-Ekkehard Matzke Cadence Design Systems GmbH, Mozartstr. 2 D-85622 Feldkirchen, Germany

e-mail: vadim@ermolayev.com, jentzsch@cadence.com, wolf@cadence.com

Frank Dengler

Institute AIFB, Karlsruhe Institute of Technology (KIT), Karlsruhe, Germany

e-mail: frank.dengler@kit.edu
} 
of the themes is the support for informal distributed process knowledge acquisition, articulation and sharing.

To this theme the notion of an informal (or knowledge) process is central. The definition in [19] lays out the ground for the specific features: Informal processes are carried out by knowledge workers with their skills, experience and knowledge, often to perform difficult tasks which require complex, informal decisions among multiple possible strategies to fulfill specific goals. In contrast to business processes which are formal, standardized, and repeatable, knowledge processes are often not even written down, let alone defined formally, vary from person to person to achieve the same objective, and are often not repeatable. Knowledge workers create informal processes on the fly in many situations of their daily work.

ACTIVE $^{1}$ has adopted a service-oriented and component-based approach to its architecture. Services and components are defined at a number of levels [19]. At the bottom level are infrastructure services. At the level above this, machine intelligence technology is used. For example the process mining service learns repeated sequences of action executions which constitute running processes and populates the knowledge base with these (Fig. 1.1). Finally at the top level are the applications. One of the case study applications is management of design project (DP) knowledge in microelectronic and integrated circuit (MIC) engineering design. This case study is lead by Cadence Design Systems $\mathrm{GmbH}^{2}$, an engineering design services provider in this domain. Cadence attempts the assessment and management of engineering design performance. This work builds on the results of $\mathrm{PSI}^{3}$ and PRODUKTIV $+{ }^{4}$ projects and goes further, beyond the existing performance management solutions by providing the functionalities of the following two kinds: (i) design process knowledge learning from distributed datasets for knowledge acquisition at the back-end; and (ii) design project knowledge articulation and sharing at the front-end - by providing a tailored lightweight collaboration platform.

Using visualized representations of artifacts in general and of processes in particular is natural for a human and requires less effort for the comprehension [1] of the logic of a process in our case.

Most process visualization techniques are included in process modeling activities, which can be centralized or decentralized. An abundance of modeling methods and tools like ARIS [16] and IDEF3 [13] have been developed to ease the standardization, storage and sharing of process visualization. Unfortu-

1 This work has been funded as part of the IST-2007-215040 EU project ACTIVE. ACTIVE - Enabling the Knowledge Powered Enterprise (http://active-project.eu/) is the EU FP7 Integrating Project.

2 http://www. cadence-europe.com/

${ }^{3}$ Performance Simulation Initiative (PSI) is the research and development project of Cadence Design Systems GmbH

${ }^{4}$ Reference System for Measuring Design Productivity of Nanoelectronic Systems (PRODUKTIV+) project has been partially funded by BMBF. 
nately these tools are not sufficient for modeling collaborative, decentralized processes. Therefore other approaches like CPM [15] have been introduced. In the area of knowledge processes additional methods and tools like KMDL [12], PROMOTE [20] and CommonKADS [17] have been developed extending the methods and tools mentioned above. In addition to that semantic wikis enable large-scale and inter-departmental collaboration on knowledge structures. Such features of semantic wikis have been extended to support process development [3] and workflows [2]. Our approach to knowledge process visualization builds upon our work on dynamic knowledge process representation projects [8]. In this our contribution is the development of the lightweight knowledge process representation for engineering design that is essentially a micro-ontology [5]. The other contribution is in process visualization - the enhancement of the existing Semantic MediaWiki (SMW) process development approach [3] for visualizing and discussing knowledge processes.

\subsection{A Solution for Design Project Knowledge Management}

In the domain of MIC engineering design a design system is denoted [10] as a holonic system ${ }^{5}$ providing the environment in which design processes are performed. Fig. 1.1 presents two particular design systems as distributed and heterogeneous environments in the bottom - one for the EU design systems based on the use of the ICD WorkBench framework and the other for the US design systems based on the use of Common Flow Initiative framework. These environments comprise (and differ in) actors, normative regulations and policies, resources, and tools. A design process executed in these environments and the environments themselves are also controlled by performance management processes comprising knowledge acquisition, measurement and assessment, analysis, decision making, and action phases [10]. A software tool for knowledge acquisition, fusion, assessment and analysis facilitates making informed decisions leading to actions that improve the performance of design systems. In the presented case study the focus is on the use and customization of the ACTIVE technologies of pro-active knowledge process support and knowledge articulation and sharing as a part of this decision making support.

There are many aspects related to engineering design process performance that require optimization [9]. One important facet is the dynamic formation of a process. Another one is the requirement to seek for the best productive process continuation among the many possible alternatives. Each execution of such a process is developed by the team of design engineers, design support

\footnotetext{
${ }^{5}$ In philosophy a holon is a system (phenomenon) that is a whole in itself as well as a part of a larger system (phenomenon).
} 
engineers and project managers in a unique fashion. It is not a challenging problem for experienced team members to make efficient follow-up decisions because they use their tacit knowledge based on experience. However, for those whose experience is limited, an expert adviser may substantially lower the risk of a mistake. It is very similar to using a navigator system for finding a route on a map, but on a map displaying design technology stages. In parallel to ACTIVE Cadence is developing such a software system for monitoring, evaluating and simulating design processes for suggesting project continuations with better performance - the ProjectNavigator [18]. At the back-end the ProjectNavigator provides a multi-agent system that simulates the execution of the DP plans by development teams. At the front-end the tool facilitates a DP manager in project planning providing intelligent support based on the knowledge incrementally collected in the Cadence Knowledge Base. This knowledge base and the tool itself are designed using the Suite of PSI Ontologies (e.g. $[8,7])^{6}$.

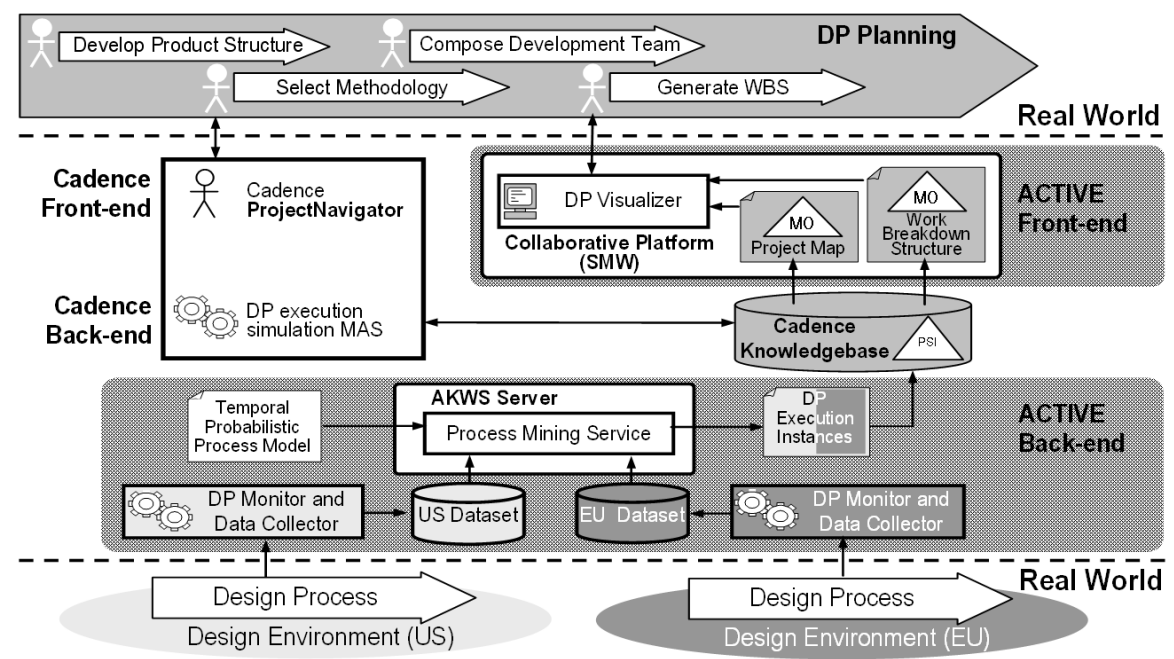

Fig. 1.1 The configuration of ACTIVE and Cadence technology components for DP knowledge acquisition, articulation and sharing.

ACTIVE technologies and software components (Fig. 1.1) are complementary and facilitating to the ProjectNavigator functionality in several important aspects: (i) at the back-end - for solving the problem of design process execution knowledge acquisition and fusion; and (ii) at the front-end - for solving the problem of DP knowledge articulation and sharing.

Acquisition is done by incremental collection of the new knowledge about the executions of design processes through monitoring design processes and

6 The documentation for the upper level of the Suite is also available as a Wiki, http: //www.kit.znu.edu.ua/mediawiki/index.php?title=PSI_Upper-Level_ontology. 
mining the dataset containing design process execution logs - using ACTIVE Process Mining component based on the probabilistic temporal process model [11]. The approach of process mining is based on the generation of the Hidden Markov Models [14]. The ACTIVated Design Framework tools monitor the design systems and the design processes and collect the data about the low level events in the respective datasets. The datasets are further fed to the Process Mining Service of the ACTIVE Knowledge WorkSpace (AKWS) Server that produces the instances of the chunks of the executed design processes in terms of the PSI Suite of Ontologies. These instances are further stored to the Cadence Knowledge Base. The fusion of the knowledge coming from distributed and heterogeneous datasets is obtained by applying the same process mining model and the same set of ontology namespaces.

Articulation and sharing are done by visualizing different facets of DP knowledge in the collaborative front-end platform using SMW - the ACTIVE DP Visualizer. Visualization functionality is structured around the typical tasks that DP managers perform in their everyday business (upper part of Fig. 1.1). The kinds of visualization pages are those for: product structures; generic methodologies; product-bound methodologies; tools; and actor roles. These primary functionalities are supported by decision making instruments for conducting moderated discussions using LiquidThreads ${ }^{7}$ extension of MediaWiki or, additionally, LiveNetLife ${ }^{8}$. ACTIVE DP Visualizer is tailored to the specific requirements of the case study. It comprises a software connector that passes the knowledge stored in the Cadence Knowledge Base into the SMW pages (Fig. 1.1).

The facts are taken from the Cadence Knowledge Base that is the repository of instances of the PSI Suite of Ontologies. The knowledge base is populated from a number of different sources. Static assertions: IP library, methodology, tool, and designer skill descriptions, are entered by human users via ProjectNavigator front-end. Process execution knowledge for the parts of the processes that have already been accomplished comes from ACTIVE process mining components. Predictions about possible continuations of the processes are generated by the ProjectNavigator back-end simulator functionality. The static knowledge, when articulated in the wiki pages, forms the basic map of the design system terrain. The mined knowledge about the executed part of a process marks the track on the terrain that the project has passed already. The simulated variants of prediction knowledge, when superimposed on the map, are essentially the suggestions of the continuations of a design process.

Knowledge workers in MIC design may be facilitated by using such an expert navigator in a variety of ways. One important use case is for making optimized project plans by exploiting the experience of the other teams in their accomplished projects. A project manager at the project planning phase is challenged by the necessity of making numerous choices of: (i) the functional

\footnotetext{
7 http://www.mediawiki.org/wiki/Extension:LiquidThreads

8 LiveNetLife is an application for contextualized interactive real-time communication between the users of a web site: http://www.livenetlife.com/.
} 
blocks from the available IP libraries to be reused; (ii) a particular sequence of technological operations that best suits the type of the developed design artifact and results in the best performance level; (iii) a most efficient and effective tool for a particular activity; (iv) the constellation of designers with matching skill sets in the development team. These choices can be made based on the analyses of the choices made in the successfully accomplished projects, especially those performed by experienced alpha teams.

Another use case exploits the synergy of the ACTIVated software and the ProjectNavigator simulation functionality. Once the project plan is detailed down to the level of a Work Breakdown Structure, it may be fed back to the ProjectNavigator simulator for exploring how it would be executed. The simulation results further articulated and shared within the development team using the DP Visualizer provide the proofs of the quality and reliability of the developed project plan.

\subsection{Validation Methodology and Results}

The software demonstrator described above implements both back-end and front-end functionalities that have been validated. The methodology for backend software validation is essentially an analytical evaluation of the components and the acquired data and knowledge. It aims at checking the quality and the completeness of data as well as the robustness of software components where applicable. The validation of the front-end part is an empirical assessment by professional users of various aspects like conformance to the requirements, appropriateness of use and usability. The majority of these validation kinds are based on the use of specific questionnaires that the users have to answer after a period of software trials. These trials are organized in the settings defined specifically for each validation kind. The feedback is collected and further analyzed. The users involved in the validation trials are Cadence DP managers also possessing significant experience as the members of development teams for various types of products. Dry runs ${ }^{9}$ have been performed leading to the refinement of the requirements to the developed software. Appropriateness and usability trials have been arranged around the four typical tasks of DP managers (upper part of Fig. 1.1). It has been observed in the preliminary interviews with the users that the most effective way to assess the utility of the demonstrator is to compare the way of executing and the results of the typical tasks in the following two cases: (i) before the introduction of ACTIVE technologies; and (ii) using the ACTIVated software. The trialists have been asked to use the DP Visualizer in the mentioned typical tasks and provide their subjective assessments in terms of the increase in performance and the decrease of the required ramp-up effort.

${ }^{9}$ A dry run is a validation trial for checking the conformance of a software artifact to the requirements. 
The trialists filled-in the specifically developed questionnaires online based on their personal assessments of the work with the offered tools.

For the front-end the dry run trials in three iterations as well as usability validation have been performed. The results for the final software revision indicate that the development is consistent with the plan. The software demonstrates reasonable conformance to the specified requirements. User perception of the demonstrator as the tool for their typical tasks is satisfactory in terms of the increase of performance. The trialists also believe that the combination of the offered technologies in the collaboration platform will facilitate to a substantial decrease of ramp-up efforts. A detailed discussion and analysis of the validation set-up and experiments are presented in [6].

\subsection{Conclusions and Future Work}

This paper presented the solution for design project knowledge articulation and sharing developed in the case study of ACTIVE project. Validation experiments proved that these functionalities are helpful for a design project manager in executing the typical tasks of project planning. The subjective assessments of the validation trialists allow believing that the introduction of the tool into everyday industrial practice may increase the performance and facilitate to decreasing the ramp-up effort of project managers and the members of their development teams.

Extending the sets of the source facts and mining more reliable assertions from these is one of the directions for our future work. Another direction is extending the visualization front-end with the functionality that generates other visualized representations of design process plans and executions. For example, the extension of the front-end that generates Gantt diagrams both

for plans and runs may allow including project execution monitoring in the sphere of the coverage of our solution.

\section{References}

1. Crapo, A.W., Waisel, L.B., Wallace, W.A., Willemain, T.R.: Visualization and the process of modeling: a cognitive-theoretic view. In: KDD '00: Proc. of the sixth ACM SIGKDD international conference on Knowledge discovery and data mining, pp. 218226. ACM, New York, NY, USA (2000)

2. Dello, K., Nixon, L., Tolksdorf, R.: Extending the makna semantic wiki to support workflows. In: Proc. of the 3rd Semantic Wiki Workshop (2008)

3. Dengler, F., Lamparter, S., Hefke, M., Abecker, A.: Collaborative process development using semantic MediaWiki. In: Proc. of the 5th Conf. of Professional Knowledge Management. Solothurn, Switzerland (2009)

4. Drucker, P.F.: The age of discontinuity : guidelines to our changing society / Peter F. Drucker. Heinemann, London (1969) 
5. Ell, B., Dengler, F., Djordjevic, D., Garzotto, F., Krtzsch, M., Siorpaes, K., Vrandecic, D., Wgler, S.: Conceptual models for enterprise knowledge final models and evaluation. Tech. Rep. D1.1.2, ACTIVE Project (2010)

6. Ermolayev, V., Jentzsch, E., Ivasiva, A., Fortuna, C., Dengler, F.: Demonstrator and validation report. Tech. Rep. D10.2.2, ACTIVE Project (2010)

7. Ermolayev, V., Jentzsch, E., Keberle, N., Sohnius, R.: Performance simulation initiative. the suite of ontologies v.2.3. reference specification. Technical Report PSI-ONTOTR-2009-1, VCAD EMEA Cadence Design Systems, GmbH (2009)

8. Ermolayev, V., Keberle, N., Matzke, W.: An upper level ontological model for engineering design performance domain. In: ER '08: Proc. of the 27th International Conf. on Conceptual Modeling, pp. 98-113. Springer-Verlag, Berlin, Heidelberg (2008)

9. Ermolayev, V., Matzke, W.: Towards industrial strength business performance management. In: HoloMAS '07: Proc. of the 3rd international conference on Industrial Applications of Holonic and Multi-Agent Systems, pp. 387-400. Springer-Verlag, Berlin, Heidelberg (2007)

10. Ermolayev, V., Matzke, W., Sohnius, R.: Engineering design performance (Invited talk). In: UNISCON, pp. 108-110 (2008)

11. Grobelnik, M., Mladenic, D., Ferlez, J.: Probabilistic temporal process model for knowledge processes: handling a stream of linked text. In: Conf. on Data Mining and Data Warehouses (SiKDD 2009). Ljubljana, Slovenia (2009)

12. Gronau, N., Mller, C., Korf, R.: KMDL - capturing, analysing and improving Knowledge-Intensive business processes. J Universal Computer Science 11(4), 452$472(2005)$

13. Mayer, R., Menzel, C., Painter, M., de Witte, P., Blinn, T., Perakath, B.: Information integration for concurrent engineering (IICE) IDEF3 process description capture method report. Tech. rep., Knowledge Based Systems Inc. (1995)

14. Rabiner, L.R.: A tutorial on hidden markov models and selected applications in speech recognition. In: Readings in speech recognition, pp. 267-296. Morgan Kaufmann Publishers Inc., San Francisco, CA, USA (1990)

15. Ryu, K., Yücesan, E.: CPM: a collaborative process modeling for cooperative manufacturers. Advanced Engineering Informatics 21(2), 231 - 239 (2007). Ontology of Systems and Software Engineering; Techniques to Support Collaborative Engineering Environments

16. Scheer, A.W., Jost, W.: ARIS in der Praxis. Springer Verlag, Berlin (2002)

17. Schreiber, G., Akkermans, H., Anjewierden, A., de Hoog, R., Shadbolt, N., van de Velde, W., Wielinga, B.: Knowledge Engineering and Management: The CommonKADS Methodology. MIT Press (1999)

18. Sohnius, R., Jentzsch, E., Matzke, W.: Holonic simulation of a design system for performance analysis. In: HoloMAS '07: Proc. of the 3rd international conference on Industrial Applications of Holonic and Multi-Agent Systems, pp. 447-454. SpringerVerlag, Berlin, Heidelberg (2007)

19. Warren, P., Kings, N., Thurlow, I., Davies, J., Bürger, T., Simperl, E., Moreno, C.R., Gomez-Perez, J., Ermolayev, V., Ghani, R., Tilly, M., Bösser, T., Imtiaz, A.: Improving knowledge worker productivity - the ACTIVE integrated approach. BT Technology Journal 26(2) (2009)

20. Woitsch, R., Karagiannis, D.: Process oriented knowledge management: A service based approach. J Universal Computer Science 11(4), 565-588 (2005) 